\title{
A NOTE ON THE LARGEST EIGENVALUE OF NON-REGULAR GRAPHS*
}

\author{
BOLIAN LIU ${ }^{\dagger}$ AND GANG LI ${ }^{\dagger}$
}

\begin{abstract}
The spectral radius of connected non-regular graphs is considered. Let $\lambda_{1}$ be the largest eigenvalue of the adjacency matrix of a graph $G$ on $n$ vertices with maximum degree $\Delta$. By studying the $\lambda_{1}$-extremal graphs, it is proved that if $G$ is non-regular and connected, then $\Delta-\lambda_{1}>\frac{\Delta+1}{n(3 n+\Delta-8)}$. This improves the recent results by B.L. Liu et al.
\end{abstract}

AMS subject classifications. 05C50, 15A48.

Key words. Spectral radius, Non-regular graph, $\lambda_{1}$-extremal graph, Maximum degree.

1. Introduction. Let $G=(V, E)$ be a simple graph on vertex set $V$ and edge set $E$, where $|V|=n$. The eigenvalues of the adjacency matrix of $G$ are called the eigenvalues of $G$. The largest eigenvalue of $G$, denoted by $\lambda_{1}(G)$, is called the spectral radius of $G$. Let $D$ denote the diameter of $G$. We suppose throughout the paper that $G$ is a simple graph. For any vertex $u$, let $\Gamma(u)$ be the set of all neighbors of $u$ and $d(u)=|\Gamma(u)|$ be the degree of $u$. A nonincreasing sequence $\pi=\left(d_{1}, d_{2}, \ldots, d_{n}\right)$ of non-negative integers is called (connected) graphic if there exits a (connected) simple graph on $n$ vertices, for which $d_{1}, d_{2}, \ldots, d_{n}$ are the degrees of its vertices. Let $\Delta$ and $\delta$ be the maximum and minimum degree of vertices of $G$, respectively. A graph is called regular if $d(u)=\Delta$ for any $u \in V$. It is easy to see that the spectral radius of a regular graph is $\Delta$ with $(1,1, \ldots, 1)^{T}$ as a corresponding eigenvector. We will use $G-e(G+e)$ to denote the graph obtained from $G$ by deleting (adding) the edge $e$. For other notations in graph theory, we follow from [2].

Stevanović [8] first found a lower bound of $\Delta-\lambda_{1}$ for the connected non-regular graphs. Then the results from [8] were improved in $[9,4,7,3]$. In $[4,7]$, the authors showed that

$$
\Delta-\lambda_{1} \geq \frac{1}{n(D+1)} \quad([4,7])
$$

\footnotetext{
${ }^{*}$ Received by the editors November 21, 2007. Accepted for publication February 15, 2008. Handling Editor: Stephen J. Kirkland.

${ }^{\dagger}$ School of Mathematics Sciences, South China Normal University, Guangzhou, 510631, P.R. China (liubl@scnu.edu.cn, tepal.li@sohu.com). This work was supported by the National Natural Science Foundation of China (No.10771080) and by DF of the Ministry of Education of China (No.20070574006).
} 
and

$$
D \leq \frac{3 n+\Delta-5}{\Delta+1} \quad([7])
$$

B.L. Liu et al. obtained

$$
\Delta-\lambda_{1} \geq \frac{\Delta+1}{n(3 n+2 \Delta-4)} \quad([7]) .
$$

Recently, S.M. Cioabă [3] improved (1.1) as follows:

$$
\Delta-\lambda_{1}>\frac{1}{n D} \quad([3]) .
$$

Thus combining (1.2) and (1.4), the inequality (1.3) can be improved as follows:

$$
\Delta-\lambda_{1}>\frac{\Delta+1}{n(3 n+\Delta-5)} .
$$

In this note we improve the inequality (1.2) on $\lambda_{1}$-extremal graphs. Furthermore, we obtain the following inequality which improves (1.5).

$$
\Delta-\lambda_{1}>\frac{\Delta+1}{n(3 n+\Delta-8)} \text {. }
$$

2. Preparation. Firstly, we state a well-known result which is just Frobenius's theorem applied to graphs.

Lemma 2.1. Let $G$ be a connected graph and $\lambda_{1}(G)$ be its spectral radius. Then $\lambda_{1}(G+u v)>\lambda_{1}(G)$ for any $u v \notin E$.

Definition 2.2. [7] Let $G$ be a connected non-regular graph. Then the graph $G$ is called $\lambda_{1}$-extremal if $\lambda_{1}(G) \geq \lambda_{1}\left(G^{\prime}\right)$ for any other connected non-regular graph $G^{\prime}$ with the same number of vertices and maximum degree as $G$.

TheOrem 2.3. Let $G$ be a $\lambda_{1}$-extremal graph on $n$ vertices with maximum degree $\Delta$. Define

$$
V_{<\Delta}=\{u: u \in V \text { and } d(u)<\Delta\} .
$$

Then $G$ must have one of the following properties:

(1) $\left|V_{<\Delta}\right| \geq 2$ and $V_{<\Delta}$ induces a complete graph.

(2) $\left|V_{<\Delta}\right|=1$.

(3) $V_{<\Delta}=\{u, v\}$, uv $\notin E(G)$ and $d(u)=d(v)=\Delta-1$.

Proof. By contradiction, suppose that $G$ is a $\lambda_{1}$-extremal graph without properties (1), (2) and (3). It follows that $\left|V_{<\Delta}\right| \geq 2$. Then there are two cases. 
Case 1: $V_{<\Delta}=\{u, v\}, u v \notin E(G), d(u)<\Delta-1$ and $d(v) \leq \Delta-1$. Then the graph $G+u v$ has the same maximum degree as $G$. By Lemma 2.1, we obtain $\lambda_{1}(G+u v)>\lambda_{1}(G)$, contradicting the choice of $G$.

Case 2: $\left|V_{<\Delta}\right|>2$ and $V_{<\Delta}$ does not induce a complete graph. Then there exist two vertices $u, v \in V_{<\Delta}$ and $u v \notin E(G)$. Similarly arguing to case 1, we obtain $\lambda_{1}(G+u v)>\lambda_{1}(G)$, contradicting the choice of $G$.

Combining the above two cases, the proof follows.

Using the properties mentioned in Theorem 2.3, we give the following definition.

Definition 2.4. Let $G$ be a connected non-regular graph on $n$ vertices with maximum degree $\Delta$. Then

the graph $G$ is called type-I if it has property (1),

the graph $G$ is called type-II if it has property (2),

the graph $G$ is called type-III if it has property (3).

Lemma 2.5. [6] Let $G$ be a simple connected graph with $n$ vertices, $m$ edges and spectral radius $\lambda_{1}(G)$. Then

$$
\lambda_{1}(G) \leq \frac{\delta-1+\sqrt{(\delta+1)^{2}+4(2 m-\delta n)}}{2}
$$

and equality holds if and only if $G$ is either a regular graph or a graph in which each vertex has degree either $\delta$ or $n-1$.

We first consider the $\lambda_{1}$-extremal graphs with $\Delta=2$ or $\Delta=n-1$. When $\Delta=2$, the $\lambda_{1}$-extremal graph is the path with $\lambda_{1}\left(P_{n}\right)=2 \cos \left(\frac{\pi}{n+1}\right)$. When $\Delta=n-1$, similarly arguing to Theorem 2.3, we know that the $\lambda_{1}$-extremal graph is $K_{n}-e$. By Lemma 2.5, we obtain

$$
\lambda_{1}\left(K_{n}-e\right)=\frac{n-3+\sqrt{(n+1)^{2}-8}}{2} .
$$

Theorem 2.3 shows that the $\lambda_{1}$-extremal graphs must be type-I, type-II or type-III, but in what follows, we will prove that when $2<\Delta<n-1$, any type-III graph is not $\lambda_{1}$-extremal.

Lemma 2.6. [5] Let $\pi=\left(d_{1}, d_{2}, \ldots, d_{n}\right)$ be a nonincreasing sequence of nonnegative integers. Then $\pi$ is graphic if and only if

$$
\sum_{i=1}^{n} d_{i} \text { is even and } \sum_{i=1}^{k} d_{i} \leq k(k-1)+\sum_{i=k+1}^{n} \min \left\{k, d_{i}\right\}, \text { for all } k=1,2, \ldots, n-1 \text {. }
$$

Lemma 2.7. Let $\pi=\left(d_{1}, d_{2}, \ldots, d_{n}\right)$ be a nonincreasing sequence of positive 
integers and $d_{n-1} \geq 2, d_{n} \geq 1$. Then $\pi$ is graphic if and only if it is connected graphic.

Proof. If $\pi$ is connected graphic, then it is obviously graphic. Conversely, suppose that $\pi$ is graphic and $G$ is a disconnected graph with the degree sequence $\pi$. Without loss of generality, suppose that $G$ has two components $G_{1}$ and $G_{2}$. Noticing $d_{n-1} \geq 2$ and $d_{n} \geq 1$, we suppose that any vertex in $G_{1}$ has degree at least two and any edge $u_{2} v_{2} \in E\left(G_{2}\right)$. Then it follows that there exists one edge $u_{1} v_{1}$ in $G_{1}$ which is not the cut edge, i.e. $G_{1}-u_{1} v_{1}$ is still connected. Otherwise, $G_{1}$ is a tree, a contradiction. Consider $G^{\prime}=G-u_{1} v_{1}-u_{2} v_{2}+u_{1} u_{2}+v_{1} v_{2}$. It is easy to see that $G^{\prime}$ is a connected graph with the degree sequence $\pi$. $\mathrm{Q}$

Lemma 2.8. Let $\pi=\left(d_{1}, d_{2}, \ldots, d_{n}\right)=(\Delta, \Delta, \ldots, \Delta, \Delta-1, \Delta-1)$ and $\pi^{\prime}=$ $\left(d_{1}^{\prime}, d_{2}^{\prime}, \ldots, d_{n}^{\prime}\right)=(\Delta, \Delta, \ldots, \Delta, \Delta-2)$ with $2<\Delta<n-1$. If $\pi$ is connected graphic, then $\pi^{\prime}$ is connected graphic.

Proof. Since $2<\Delta<n-1$, we obtain $d_{n-1}^{\prime} \geq 3$ and $d_{n}^{\prime} \geq 1$. Then by Lemma 2.7 , we need only to prove that $\pi^{\prime}$ is graphic. Let $G$ be a connected graph with the degree sequence $\pi$. Since $\pi$ is graphic, by Lemma 2.6 , we obtain

$$
\sum_{i=1}^{n} d_{i} \text { is even and } \sum_{i=1}^{k} d_{i} \leq k(k-1)+\sum_{i=k+1}^{n} \min \left\{k, d_{i}\right\}, \text { for all } k=1,2, \ldots, n-1 .
$$

For $\pi^{\prime}$ we will prove that (2.2) is still true. Obviously, $\sum_{i=1}^{n} d_{i}^{\prime}=\sum_{i=1}^{n} d_{i}$ is even. Then we need only to prove that the inequality is true. We split our proof into four cases.

Case 1: $1 \leq k \leq \Delta-2$, then $k \leq n-4$ and

$$
\begin{aligned}
\sum_{i=1}^{k} d_{i}^{\prime} & =\sum_{i=1}^{k} d_{i} \leq k(k-1)+\sum_{i=k+1}^{n} \min \left\{k, d_{i}\right\} \\
& =k(k-1)+k(n-k) \\
& =k(k-1)+\sum_{i=k+1}^{n} \min \left\{k, d_{i}^{\prime}\right\} .
\end{aligned}
$$

Case 2: $1<k=\Delta-1$, then $k \leq n-3$ and

$$
k(k-1)+k(n-k)-\sum_{i=1}^{k} d_{i}=k(k-1)+k(n-k)-k \Delta=k(n-k-2) \geq k>1 .
$$

Thus

$$
\sum_{i=1}^{k} d_{i}^{\prime}=\sum_{i=1}^{k} d_{i}<k(k-1)+k(n-k)-1=k(k-1)+\sum_{i=k+1}^{n} \min \left\{k, d_{i}^{\prime}\right\} .
$$


Case 3: $\Delta \leq k \leq n-2$, then

$$
\begin{aligned}
\sum_{i=1}^{k} d_{i}^{\prime}=\sum_{i=1}^{k} d_{i} & \leq k(k-1)+\sum_{i=k+1}^{n} \min \left\{k, d_{i}\right\} \\
& =k(k-1)+(n-k-2) \Delta+2 \Delta-2 \\
& =k(k-1)+\sum_{i=k+1}^{n} \min \left\{k, d_{i}^{\prime}\right\} .
\end{aligned}
$$

Case 4: $k=n-1$, then

$$
\begin{aligned}
k(k-1)+\Delta-\sum_{i=1}^{k} d_{i} & =(n-1)(n-2)+\Delta-[(n-1) \Delta-1] \\
& =(n-2)(n-1-\Delta)+1 \geq 4,
\end{aligned}
$$

where the last inequality holds since $2<\Delta<n-1$. Hence

$$
\begin{aligned}
\sum_{i=1}^{k} d_{i}^{\prime} & =\sum_{i=1}^{k} d_{i}+1<k(k+1)+\Delta-2 \\
& =k(k-1)+\sum_{i=k+1}^{n} \min \left\{k, d_{i}^{\prime}\right\} .
\end{aligned}
$$

Combining the above four cases, the inequality is true. Then by Lemma 2.6, the $\pi^{\prime}$ is graphic. This completes the proof.

As we know, majorization on degree sequences is defined as follows: for two sequences $\pi=\left(d_{1}, d_{2}, \ldots, d_{n}\right), \pi^{\prime}=\left(d_{1}^{\prime}, d_{2}^{\prime}, \ldots, d_{n}^{\prime}\right)$ we write $\pi \unlhd \pi^{\prime}$ if and only if $\sum_{i=1}^{n} d_{i}=\sum_{i=1}^{n} d_{i}^{\prime}$ and $\sum_{i=1}^{j} d_{i} \leq \sum_{i=1}^{j} d_{i}^{\prime}$ for all $j=1,2, \ldots, n$. We claim that $G$ has the greatest maximum eigenvalue if $\lambda_{1}(G) \geq \lambda_{1}\left(G^{\prime}\right)$ for any other graph $G^{\prime}$ in the class $\mathcal{C}_{\pi}$, where $\mathcal{C}_{\pi}=\{G: G$ is a connected graph with the degree sequence $\pi\}$.

Lemma 2.9. [1] Let $\pi$ and $\pi^{\prime}$ be two distinct degree sequences with $\pi \unlhd \pi^{\prime}$. Let $G$ and $G^{\prime}$ be graphs with the greatest maximum eigenvalues in classes $\mathcal{C}_{\pi}$ and $\mathcal{C}_{\pi^{\prime}}$, respectively. Then $\lambda_{1}(G)<\lambda_{1}\left(G^{\prime}\right)$.

TheOREM 2.10. Let $G$ be a connected graph with degree sequence

$$
\pi=(\Delta, \Delta, \ldots, \Delta, \Delta-1, \Delta-1)
$$

and $2<\Delta<n-1$. Then there exists a connected graph $G^{\prime}$ with degree sequence $\pi^{\prime}=(\Delta, \Delta, \ldots, \Delta, \Delta-2)$ such that $\lambda_{1}(G)<\lambda_{1}\left(G^{\prime}\right)$.

Proof. By Lemma 2.8, there exists a connected graph $G^{\prime}$ with degree sequence $\pi^{\prime}=(\Delta, \Delta, \ldots, \Delta, \Delta-2)$. We suppose that $G\left(G^{\prime}\right)$ is the graph with the greatest 
maximum eigenvalue in $\mathcal{C}_{\pi}\left(\mathcal{C}_{\pi^{\prime}}\right)$. It is obvious that $\pi \unlhd \pi^{\prime}$. By Lemma 2.9, we obtain $\lambda_{1}(G)<\lambda_{1}\left(G^{\prime}\right)$.

TheOrem 2.11. Let $G$ be a $\lambda_{1}$-extremal graph on $n$ vertices with the maximum degree $\Delta$ and $2<\Delta<n-1$. Then $G$ must be either type-I or type-II.

Proof. Suppose that $G$ is a type-III graph with the greatest maximum eigenvalue in class $\mathcal{C}_{\pi}$, where $\pi=(\Delta, \Delta, \ldots, \Delta, \Delta-1, \Delta-1)$. By Theorem 2.10, there exists a graph $G^{\prime}$ with degree sequence $\pi^{\prime}=(\Delta, \Delta, \ldots, \Delta, \Delta-2)$ and greatest maximum eigenvalue in class $\mathcal{C}_{\pi}^{\prime}$ such that $\lambda_{1}\left(G^{\prime}\right)>\lambda_{1}(G)$. It follows that $G$ is not $\lambda_{1}$-extremal.

Remark. Although Theorem 2.11 shows that the $\lambda_{1}$-extremal graph with $2<$ $\Delta<n-1$ must be type-I or type-II, there exist some graphs with property (1) or (2) which are not $\lambda_{1}$-extremal. Let $G_{1}, G_{2}$ be connected graphs with degree sequences $(3,3,3,3,2,2),(5,5,5,5,5,5,2)$, respectively. Clearly $G_{1}\left(G_{2}\right)$ is a type-I (type-II) graph. However, by checking the Table 1 of [7], we know they are not the $\lambda_{1}$-extremal graphs. After some computer experiments, we give a conjecture about the $\lambda_{1}$-extremal graphs as follows:

CONJECTURE 2.12. Let $G$ be a connected non-regular graph on $n$ vertices and $2<\Delta<n-1$. Then $G$ is $\lambda_{1}$-extremal if and only if $G$ is a graph with the greatest maximum eigenvalue in classes $\mathcal{C}_{\pi}$ and $\pi=(\Delta, \Delta, \ldots, \Delta, \delta)$, where

$$
\delta= \begin{cases}(\Delta-1), & \text { when } n \Delta \text { is odd } \\ (\Delta-2), & \text { when } n \Delta \text { is even } .\end{cases}
$$

\section{Main Results.}

TheOREm 3.1. Let $G$ be a type-I or type-II graph on $n$ vertices with diameter $D$. Then

$$
D \leq \frac{3 n+\Delta-8}{\Delta+1}
$$

Proof. Since $G$ is a type-I or type-II graph, we have $\Delta \geq 3$. Let $u, v$ be two vertices at distance $D$ and $P: u=u_{0} \leftrightarrow u_{1} \leftrightarrow \ldots \leftrightarrow u_{D}=v$ be the shortest path connecting $u$ and $v$. We first claim that $\left|V_{<\Delta} \cap V(P)\right| \leq 2$. Otherwise, $G$ must be a type-I graph and suppose $\left\{u_{p}, u_{q}, u_{r}\right\} \subseteq V(P) \cap V_{<\Delta}$ with $p<q<r$. Then by definition of type-I graph, we obtain that $u_{p} u_{q}, u_{q} u_{r}$ and $u_{p} u_{r} \in E(G)$. Therefore, $P$ is not the shortest path connecting $u$ and $v$, a contradiction.

Then there are two cases.

Case 1: $V_{<\Delta} \cap V(P)=\varnothing$. Define $T=\{i: i \equiv 0 \bmod 3$ and $i \leq(D-3)\} \bigcup\{D\}$. Thus $|T|=\left\lceil\frac{D+1}{3}\right\rceil$. Let $d\left(u_{i}, u_{j}\right)$ denote the distance between $u_{i}$ and $u_{j}$. Since $P$ is 
the shortest path connecting $u$ and $v$, we have $d\left(u_{i}, u_{j}\right) \geq 3$ and $\Gamma\left(u_{i}\right) \cap \Gamma\left(u_{j}\right)=\varnothing$ for any distinct $i, j \in T$. Notice that $u_{i} \in V(P)$ for any $i \in T$. We obtain

$$
\left|\Gamma\left(u_{i}\right)-V(P)\right|= \begin{cases}\Delta-1, & \text { if } i \in\{0, \mathrm{D}\} \\ \Delta-2, & \text { otherwise }\end{cases}
$$

Then

$$
\begin{aligned}
n & \geq|V(P)|+\sum_{i \in T}\left|\Gamma\left(u_{i}\right)-V(P)\right| \\
& \geq D+1+(|T|-2)(\Delta-2)+2(\Delta-1) \\
& \geq D+1+\left(\frac{D+1}{3}-2\right)(\Delta-2)+2(\Delta-1) .
\end{aligned}
$$

Thus

$$
D \leq \frac{3 n-\Delta-7}{\Delta+1}
$$

Case 2: Either $V_{<\Delta} \cap V(P)=\left\{u_{p}, u_{q}\right\}$ with $q=p+1$ or $V_{<\Delta} \cap V(P)=\left\{u_{p}\right\}$. The proof is similar to the proof of [7]. We obtain the same result

$$
D \leq \frac{3 n+\Delta-8}{\Delta+1}
$$

Combining the above two cases, the proof follows. $\mathrm{Q}$

Lemma 3.2. [3] Let $G$ be a connected non-regular graph on $n$ vertices with maximum degree $\Delta$ and diameter $D$. Then

$$
\Delta-\lambda_{1}>\frac{1}{n D}
$$

TheOREM 3.3. Let $G$ be a connected non-regular graph on $n$ vertices with maximum degree $\Delta$. Then

$$
\Delta-\lambda_{1}>\frac{\Delta+1}{n(3 n+\Delta-8)} .
$$

Proof. Without loss of generality, we suppose that $G$ is a $\lambda_{1}$-extremal graph. Since $G$ is connected and non-regular, then $n \geq 3$ and $\Delta \geq 2$. When $\Delta=n-1$ and $n \geq 5$, the $\lambda_{1}$-extremal graph is $K_{n}-e$ with $D=2$. Then by Lemma 3.2 , we obtain

$$
\lambda_{1}\left(K_{n}-e\right)<\Delta-\frac{1}{2 n}<\Delta-\frac{\Delta+1}{n(3 n+\Delta-8)} .
$$


When $\Delta=n-1$ and $n=3$, the $\lambda_{1}$-extremal graph is $P_{3}$ with $\lambda_{1}\left(P_{3}\right)=1.4142$. When $\Delta=n-1$ and $n=4$, the $\lambda_{1}$-extremal graph is $K_{4}-e$ with $\lambda_{1}\left(K_{4}-e\right)=2.5616$. By direct calculation, we know that the inequality is true. When $2<\Delta<n-1$, applying Theorem 3.1 and Lemma 3.2, we obtain the result. When $\Delta=2$ and $n>3$, the $\lambda_{1}$-extremal graph is $P_{n}$. By adding some edges to $P_{n}$, we can attain $K_{n}-e$. Then following the Lemma 2.1, we obtain $\lambda_{1}\left(P_{n}\right)<\lambda_{1}\left(K_{n}-e\right)$. This completes the proof.

Acknowledgement. The authors would like to thank an anonymous referee for valuable comments and suggestions that improved our presentation.

\section{REFERENCES}

[1] T. Biyikoglu and J. Leydold. Largest eigenvalue of degree sequences. Available at http://arxiv.org/abs/math.CO/0605294.

[2] J.A. Bondy and U.S.R. Mury. Graph Theory with Application. North-Holland, New York, 1976.

[3] S.M. Cioabă. The spectral radius and the maximum degree of irregular graphs. Electron. J. Combin., 14 (2007), R38.

[4] S.M. Cioabă, D.A. Gregory, and V. Nikiforov. Note: extreme eigenvalues of nonregular graphs. J. Combin. Theory Ser. B, 97:483-486, 2007.

[5] P. Erdös and T. Gallai. Graphs with prescribed degree of vertices (in Hungarian). Mat. Lapok 11, 1960.

[6] Y. Hong, J.L. Shu, and K. Fang. A sharp upper bound of the spectral radius of graphs. J. Combin. Theory Ser. B, 81:177-183, 2001.

[7] B.L. Liu, J. Shen, and X.M. Wang. On the largest eigenvalue of non-regular graphs. J. Combin. Theory Ser. B, 97:1010-1018, 2007.

[8] D. Stevanović. The largest eigenvalue of nonregular graphs. J. Combin. Theory Ser. B, 91:143$146,2004$.

[9] X.D. Zhang. Eigenvectors and eigenvalues of non-regular graphs. Linear Algebra Appl., 409:7986, 2005. 